\title{
Filamin A and FILIP (Filamin A-Interacting Protein) Regulate Cell Polarity and Motility in Neocortical Subventricular and Intermediate Zones during Radial Migration
}

\author{
Takashi Nagano, ${ }^{1}$ Soichi Morikubo, ${ }^{1,2}$ and Makoto Sato ${ }^{1,3}$ \\ ${ }^{1}$ Division of Cell Biology and Neuroscience, Department of Morphological and Physiological Sciences, and ${ }^{2}$ Division of Ophthalmology, Department of \\ Sensory and Locomotor Medicine, Faculty of Medical Sciences, University of Fukui, Matsuoka, Fukui 910-1193, Japan, and ${ }^{3}$ Solution Oriented Research for \\ Science and Technology, Japan Science and Technology Corporation, Kawaguchi, Saitama 332-0012, Japan
}

In the developing neocortex, most excitatory neurons are supplied and arranged through radial migration. Because neurons show global morphological changes and complicated behavior during that migration, precise regulation of cell shape and polarity is essential for proper migration and correct neocortical formation; however, how cell shape and polarity are regulated in migrating neuron remains elusive. We show here that Filamin A, a well known actin-binding protein, determines the shape of neocortical neurons during radial migration in vivo. Dysfunction of Filamin A, caused by a mutant Filamin A expression, prevents cells from acquiring consistent polarity toward specific direction and decreases motility in the subventricular and intermediate zones. In contrast, Filamin A overexpression, achieved by a short interfering RNA for Filamin A-interacting protein that induces Filamin A degradation (FILIP), promotes the development and maintenance of a bipolar shape also in the subventricular and intermediate zones. These results suggest that the amount of Filamin A helps migrating neurons determine their mode of migration, multipolar or bipolar, before entering the cortical plate and that FILIP is responsible, at least in part, for Filamin A content. In addition, our results also give a possible clue to understanding the pathogenesis of human malformation periventricular heterotopia, which is caused by various "loss-of-function" mutations in the filamin $A$ gene.

Key words: cortex; migration; multipolar migration; actin; cytoskeleton; periventricular heterotopia

\section{Introduction}

Radial migration in the neocortex, during which neurons travel from the ventricular zone (VZ) to the cortical plate (CP) exhibiting varying morphology and motility (Rakic, 1972; Miyata et al., 2001; Nadarajah et al., 2001), is an essential process in corticogenesis. In particular, studies have been focused on the complex behavior of migrating neurons in the subventricular and intermediate zones (SVZ-IZ). For example, Tabata and Nakajima (2003) have observed abundant multipolar cells in this region; these show slow, irregular movement together with dynamic extension and retraction of their multiple neurites. Noctor et al. (2004) described four distinct phases that migrating neurons un-

\footnotetext{
Received June 16, 2004; revised Aug. 18, 2004; accepted Sept. 10, 2004.

This work was supported in part by the 21st Century COE program (Biomedical Imaging Technology Integration Program), the Toray Science Foundation, the Uehara Memorial Foundation, and the Ministry of Education, Culture, Sports, Science and Technology of Japan. We thank Y. Hatanaka and D. Konno for technical advice; H. Yoshikawa, N Kato, S. Gunjima, and N. Shimada for their technical assistance; H. Yagi and K. Ando for helpful discussions; and Y Akagi for encouragement. We are also grateful to J. Miyazaki for pGAGGS vector, J. Y. Yu and D. L. Turner for mU6pro vector, and T. P. Stossel and Y. Ohta for the human Filamin A CDNA.

Correspondence should be addressed to M. Sato, Division of Cell Biology and Neuroscience, Department of Morphological and Physiological Sciences, Faculty of Medical Sciences, University of Fukui, Matsuoka, Fukui 9101193, Japan. E-mail: makosato@fmsrsa.fukui-med.ac.jp.

D0I:10.1523/JNEUROSCI.2363-04.2004

Copyright $\odot 2004$ Society for Neuroscience $\quad 0270-6474 / 04 / 249648-10 \$ 15.00 / 0$
}

dergo before entering the $\mathrm{CP}$, during which they halt and reverse direction. These observations suggest that radial migration through the SVZ-IZ is not simply a process to deliver neurons to the CP but has unexpected significance in regulating and/or coordinating corticogenesis through cellular interaction. Therefore, how these morphological changes and complex behavior are regulated is an important issue for understanding corticogenesis.

Hereditary neocortical malformations that result from aberrant radial migration could provide important clues to understanding the molecular mechanisms that regulate cell behavior during migration. Several essential proteins, such as Lis1 and Doublecortin, have actually been identified through analyses of various malformations (Gupta et al., 2002). Interestingly, such proteins are related to microtubule dynamics (Feng et al., 2000; Smith et al., 2000; Schaar et al., 2004; Tanaka et al., 2004), indicating that reorganization of the microtubule is crucial for radial migration. However, because the microtubule is known to be involved in neuronal migration primarily through intracellular nuclear translocation (Rakic et al., 1996; Feng et al., 2000; Smith et al., 2000; Xie et al., 2003), how cell shape and polarity are regulated in migrating neuron remains elusive.

In this study, we provide evidence indicating that Filamin $\mathrm{A}$, a well known actin-binding protein, is involved in regulating cell 
polarity and motility in the SVZ-IZ during radial migration. Because various "loss-of-function" mutations in human filamin $A$ gene cause periventricular heterotopia in which cortical neurons accumulate beside the lateral ventricle (Eksioglu et al., 1996; Fox et al., 1998; Sheen et al., 2001; Kakita et al., 2002), it has been suggested that Filamin A is required for the early phases of radial migration (e.g., radial migration start). However, the precise nature of its involvement is still an open question. We investigated this issue using a mutant Filamin A molecule and a short interfering RNA (siRNA) for Filamin A-interacting protein (FILIP) that induces Filamin A degradation (Nagano et al., 2002) to inhibit and enhance, respectively, the activity of Filamin A. Our results show that cells with suppressed Filamin A function have difficulty in acquiring consistent polarity and establishing a bipolar form in the SVZ-IZ, whereas Filamin A overexpression increases the number of bipolar cells. These results suggest that Filamin A is essential for regulating the polarity of neocortical neurons during radial migration and that the actin cytoskeleton (on which Filamin A resides), as well as the microtubule cytoskeleton, is also important for radial migration as shown in the cerebellum (Rivas and Hatten, 1995).

\section{Materials and Methods}

Animals. Pregnant Wistar rats and C57BL/6 mice were purchased from a local vendor (SLC, Hamamatsu, Japan), housed at a constant temperature and humidity, and provided with food and water ad libitum. Embryonic day 0 (E0) was defined as the day of confirmation of the vaginal plug. All pregnant animals were deeply anesthetized by intraperitoneal injection of 2,2,2-Tribromoethanol (200-300 mg/kg body weight) before the experiments. All experiments were conducted in compliance with the Guidelines for Use of Laboratory Animals of University of Fukui, and all efforts were made to minimize the number of animals used and their suffering.

Plasmid vectors and antibodies. For protein expression, plasmid vectors constructed on pGAGGS (Niwa et al., 1991) were used so that the cDNAs were driven under a CAG promoter. For siRNA expression, plasmid vectors constructed on mU6pro (Yu et al., 2002) were used so that the siRNAs were expressed under a mouse U6 promoter (see below). The following antibodies were used: anti-green fluorescent protein (GFP) antibody (\#632377; Clontech, Palo Alto, CA), anti-Filamin A antibody (sc-17749; Santa Cruz Biotechnology, Santa Cruz, CA), a customized anti-Filamin A antibody (Sigma Genosys Japan, Ishikari, Japan) raised against a synthetic peptide corresponding to residues $1742-1755$ of mouse Filamin A protein (SWISS-PROT Q8BTM8), anti- $\beta$ III-tubulin antibody (TuJ1; PRB-435P; Covance Research Products, Berkeley, CA), Alexa Fluor 568-labeled anti-rabbit IgG antibody (A11011; Molecular Probes, Eugene, OR), and anti-actin antibody (A4700; Sigma, St. Louis, MO).

Electroporation-mediated gene transfer and culture of the neocortical explants. The methods used for DNA transfection and culture of the neocortical explants have been described previously (Hatanaka and $\mathrm{Mu}$ rakami, 2002). In brief, $\sim 2 \mu \mathrm{l}$ of plasmid DNA $(0.5-5 \mu \mathrm{g} / \mu \mathrm{l})$ was injected into the lateral ventricle of E18 rat brain and then introduced into the ventricular zone cells by delivering five electrical pulses ( $50 \mathrm{~V} ; 50$ msec duration) at intervals of $75 \mathrm{msec}$ with a square-pulse electroporator (CUY21EDIT; Nepa Gene, Ichikawa, Japan) and a tweezers-type electrode with disc electrodes ( $5 \mathrm{~mm}$ in diameter) at the tip (CUY650-5; Nepa Gene). For cotransfection, a molar ratio of 1 [enhanced GFP (EGFP)-expression plasmid] to - 10 (siRNA expression plasmid) was used. The brain was cut coronally into $200 \mu \mathrm{m}$ slices with a Vibratome (VT1000S; Leica Microsystems, Wetzlar, Germany), and the dorsolateral portion of the cortex was dissected out and cultured on a collagen-coated membrane (Transwell-COL; Costar-Corning, Corning, NY) in DMEM/ F12 medium containing 10\% fetal bovine serum and N2 supplement. For analyzing migration or cell shape, the sections were fixed with $4 \%$ paraformaldehyde $/ 0.1 \mathrm{~m}$ phosphate buffer, $\mathrm{pH} 7.4$, for $30 \mathrm{~min}$ and imaged on a laser-scanning confocal microscope (LSM 5 PASCAL; Carl-Zeiss,
Oberkochen, Germany). The migration was quantified based on the cortical location in which its cell body was situated in three independent experiments (three slices; $>200$ cells per slice) in each group. For cell shape analysis, radially oriented spindle-shaped cells (defined as those with a long axis/short axis ratio of $>2.5$ ) were identified within $100 \mu \mathrm{m}$ from the ventricular surface, and their frequency among the total number of labeled cells in the same region was calculated on three independent experiments (three slices; $30-92$ cells per slice) in each group. Because the edges of the explants (especially the ventricular surfaces) gradually deform during culture period, the locations of original ventricular surfaces were estimated based on some cells with EGFP labeling that hardly migrate in the VZ. For protein analysis, the sections were detached from the membranes, lysed, and subjected to immunoblotting.

RNA interference. Three different constructs for FILIP siRNAs were prepared on the mU6pro vector (Yu et al., 2002). These expressed the short hairpin RNAs 5'-GUGGACUUCGAAGACAAGGCCuucaa-

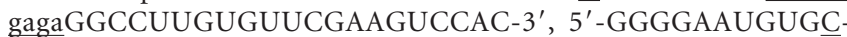
AAACUCCAGUuucaagagaACUGGAGUUUCCACAUUCCCC-3' and 5 '-GCGCGUGCUUCAGAUGGAGGGuucaagagaCCCUCCAUCUCAAGCACGCGC-3' to target nucleotides 972-992, 1176-1196, and 1226-1246 of rat L-FILIP cDNA (GenBank accession number NM145682) (L-FILIP is one of the two known FILIP species) and were termed FILIP siRNA-1, -2, and -3, respectively. The underlined single nucleotides represent the mutations introduced, whereas the underlined sequences in lower case correspond to the loop region. These targets are common to L- and S-FILIP cDNAs (S-FILIP is another known FILIP species), and identical nucleotide sequences for each target are also found in mouse putative FILIP cDNA (GenBank accession number AK017709), 93\% of the corresponding nucleotides of which are identical to those of rat L-FILIP cDNA. To assess the effects of these siRNAs, an expression vector for S-FILIP-EGFP was cotransfected into COS-7 cells with each of the siRNA-expressing vector or mU6pro vector, and the amount of S-FILIP-EGFP in the cells was compared by immunoblotting.

In utero electroporation-mediated gene transfer, tissue preparation, and immunohistochemistry. The method was performed essentially as described previously (Saito and Nakatsuji, 2001; Tabata and Nakajima, 2001). Briefly, pregnant mice were deeply anesthetized on E15, and the uterine horns were exposed. Plasmid DNA, purified using the EndoFree plasmid kit (Qiagen, Hilden, Germany), was dissolved in HBS (21 mM HEPES, pH 7.0, $137 \mathrm{~mm} \mathrm{NaCl}, 5 \mathrm{~mm} \mathrm{KCl}, 0.7 \mathrm{~mm} \mathrm{Na} \mathrm{HPO}_{4}, 1 \mathrm{mg} / \mathrm{l}$ glucose) at a final concentration of $0.5-3.5 \mu \mathrm{g} / \mu \mathrm{l}$ together with Fast Green (final concentration $0.01 \%$ ). For cotransfection, a molar ratio of 1 (EGFP-expression plasmid) to 3-6 (the other plasmids for cotransfection) was used. Approximately $2 \mu \mathrm{l}$ of plasmid solution was injected into the lateral ventricle from outside the uteri with a glass micropipette (GD1.5 , Narishige, Tokyo, Japan). Each embryo in the uterus was then placed between the tweezers-type electrodes described above and electronic pulses ( $40 \mathrm{~V} ; 50 \mathrm{msec}$ duration) were applied five times at intervals of 950 $\mathrm{msec}$. The uterine horns were then placed back into the abdominal cavity to allow the embryos to continue normal development.

Two or $4 \mathrm{~d}$ after electroporation (E17 or 19, respectively), the pregnant dams were killed, and the brains of the embryos were fixed by overnight immersion (for E17 brains) or transcardial perfusion followed by overnight immersion (for E19 brains) using the same fixative as described above for the neocortical explants. For analyzing cell shape and migration, $100-\mu \mathrm{m}$-thick sections were made on a Vibratome. For immunohistochemistry, $14-\mu \mathrm{m}$-thick frozen sections were made on a cryostat and subjected to antibody reaction. The sections at the anterior one-third of the neocortex were imaged and analyzed on a laser-scanning confocal microscope. The migration was quantified based on the cortical location in which its cell body was situated in three independent experiments (three slices; $48-234$ cells per slice) in each group. For cell shape analysis, radially oriented spindle-shaped cells (defined as those with a long axis/ short axis ratio of $>2.5$ ) were identified, and their frequency among the total number of labeled cells in the same region was calculated on three independent experiments (three slices; 21-64 cells per slice) in each group.

Time-lapse observation. Two days after in utero electroporation- 
mediated gene transfer on E15, the pregnant dams were killed, and the embryos (E17) were taken out. Coronal slices of the embryonic brain (200 $\mu \mathrm{m}$ thick) were made on a Vibratome, and slices from the anterior one-third of the neocortex were placed on a glass-bottomed dish and mounted in collagen gel. These were cultured in the same medium as the neocortical explants in a $\mathrm{CO}_{2}$ incubation chamber $\left(5 \% \mathrm{CO}_{2}\right.$ at $\left.37^{\circ} \mathrm{C}\right)$ that was fitted onto a confocal microscope. The dorsolateral region of the neocortex was analyzed by automatically obtaining $20-30$ optical Z sections every $20 \mathrm{~min}$ for $7-15 \mathrm{hr}$.

Statistical analyses. Significant differences, with $p<0.05$, were determined by $t$ test.

\section{Results}

Filamin A that lacks the actin-binding domain alters the shape of neocortical neurons during migration in organotypic explants

We used a Filamin A mutant without the actin-binding domain ( $\triangle \mathrm{ABD}$-Filamin A) to suppress Filamin A activity. Because Filamin $\mathrm{A}$ is known to form a signaling scaffold with various other proteins as well as with $\mathrm{F}$-actin through its actin-binding domain (Stossel et al., 2001), it is likely that $\Delta \mathrm{ABD}$-Filamin A interferes with Filamin A functions to link the signaling scaffold with F-actin. Actually, Kainulainen et al. (2002) reported that $\Delta \mathrm{ABD}$ Filamin A prevents the mechanoprotective function of Filamin A. To assess the significance of Filamin A during neuronal migration, we used electroporation-mediated gene transfer to express the mutant human Filamin A in E18 rat neocortical neurons, which migrate radially in brain explants, as reported previously (Hatanaka and Murakami, 2002; Nagano et al., 2002). Briefly, expression plasmids were injected into the lateral ventricle, and electrical pulses were applied so that the plasmids were incorporated into the cells of the VZ. The transfected cortices were then dissected out, sliced, and cultured. Using this method, some of the postmitotic neurons were induced to overexpress EGFPtagged mutant Filamin A (EGFP- $\triangle \mathrm{ABD}$-Filamin A) or EGFP only (control). After $5 \mathrm{~d}$ of culture, many cells expressing control EGFP exhibited the radially oriented spindle shape with leading and trailing processes that are characteristic of migrating neurons (Fig. $1 A$, left column). In contrast, those showing intense EGFP$\triangle \mathrm{ABD}$-Filamin $\mathrm{A}$ expression were oval or round in shape and their migration was suppressed, whereas those containing less EGFP- $\triangle \mathrm{ABD}$-Filamin A showed apparently normal morphology and motility (Fig. $1 A$, right column, $B$ ), suggesting that the mutant exerted a dose-dependent inhibitory effect against radial migration. Therefore, the shape of the neocortical neurons, as well as their motility during radial migration, appears to depend on Filamin A.

\section{FILIP and Filamin A determine the shape of neocortical neurons in organotypic explants}

To confirm the significance of Filamin A in determining the shape of neocortical neurons in the explants, we next attempted to enhance the function of endogenous Filamin A by suppressing S- and L-FILIP (Nagano et al., 2002). We used FILIP siRNA, which was expressed using a plasmid vector (Yu et al., 2002). First, we constructed three vectors, each of which expressed a distinct FILIP siRNA (FILIP siRNA-1-siRNA-3), and each vector was transfected into COS-7 cells together with an expression vector for S-FILIP-EGFP. The effects of the siRNAs were compared by quantifying the S-FILIP-EGFP protein. We found that FILIP siRNA-3 was the most effective, whereas FILIP siRNA-2 had little effect (Fig. 2A). The FILIP siRNA-3 expression vector was therefore coelectroporated with an EGFP expression vector into E18 rat $\mathrm{VZ}$ cells, and the transfected cortices were maintained as or-
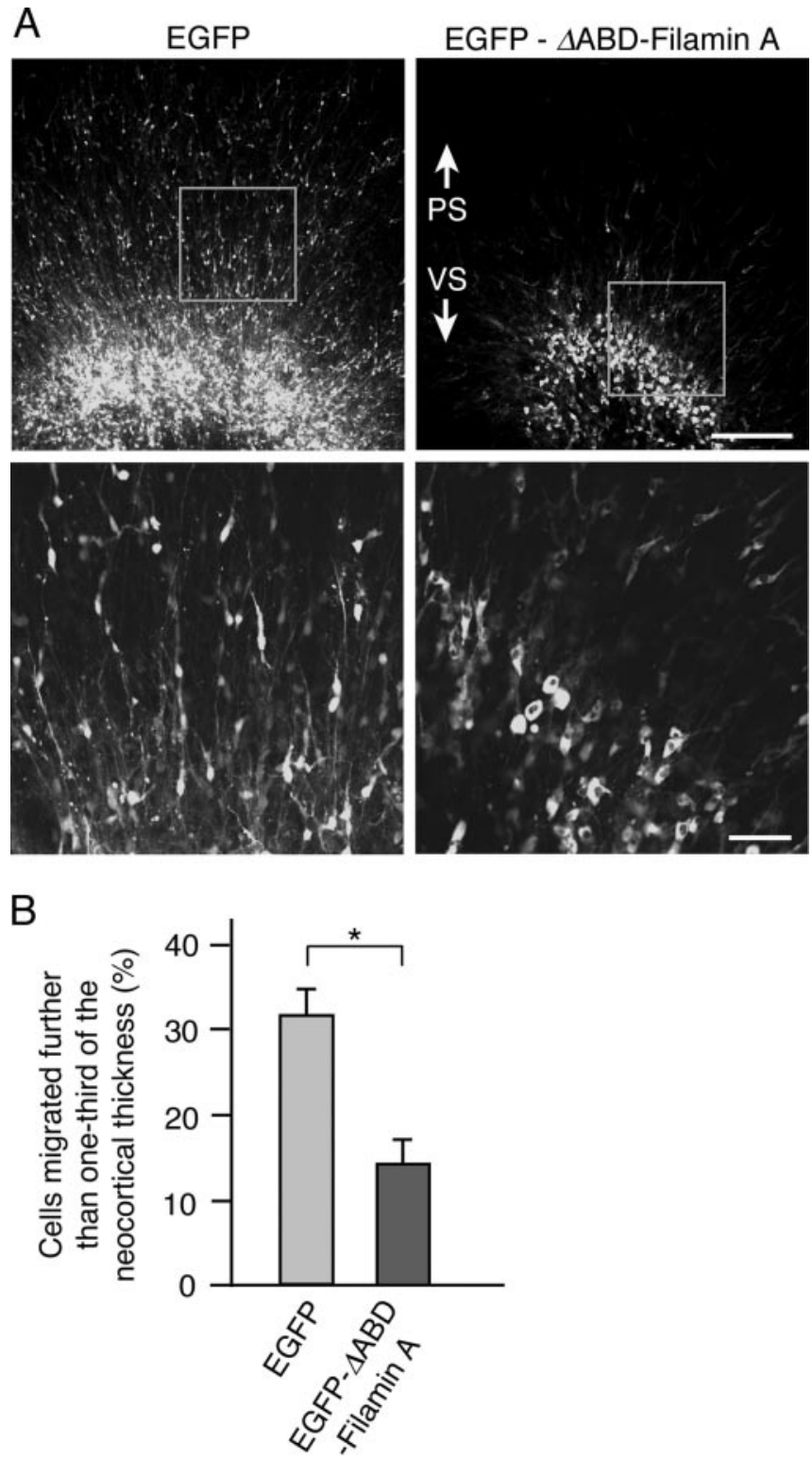

Figure 1. $\triangle \mathrm{ABD}$-Filamin $\mathrm{A}$ inhibits radial migration in cultured neocortical explants. $A$, CDNAs of EGFP (left column) or EGFP- $\triangle$ ABD-Filamin A (right column) were electroporatically introduced into the cells of the ventricular zone of the neocortex in E18 rats. These cells were then maintained as explants for $5 \mathrm{~d}$. A substantial number of the EGFP-expressing cells were spindle shaped and migrated radially toward the pial side (PS), whereas most cells showing intense EGFP- $\triangle$ ABD-Filamin A expression were more dumpy or round in shape and remained close to the ventricular surface (VS). The bottom panels show higher-magnification views of the areas indicated by the squares in the top panels. Scale bars: top panels, $200 \mu \mathrm{m}$; bottom panels, $50 \mu \mathrm{m}$. B, Cell motility in $A$ was assessed by calculating the percentage of cells that migrated farther than one-third of the neocortical thickness within the explants. The mean and SEM for three independent experiments are shown for each group. The asterisk indicates a significant difference between the groups ( $p<0.05 ; t$ test).

ganotypic explants as described above. This coelectroporation generally enabled both vectors to be introduced into the same cell (data not shown). As expected, introduction of FILIP siRNA-3 caused excessive storage of Filamin A in the explants (Fig. $2 \mathrm{~B}$, middle lane), confirming that the siRNA was effective and agreeing with the view that FILIP acts to induce the degradation of Filamin A. Then we assessed the morphology of the cells expressing FILIP siRNA-3 (together with EGFP) after $2 \mathrm{~d}$ of culture, when most of the cells carrying the transfected plasmids re- 


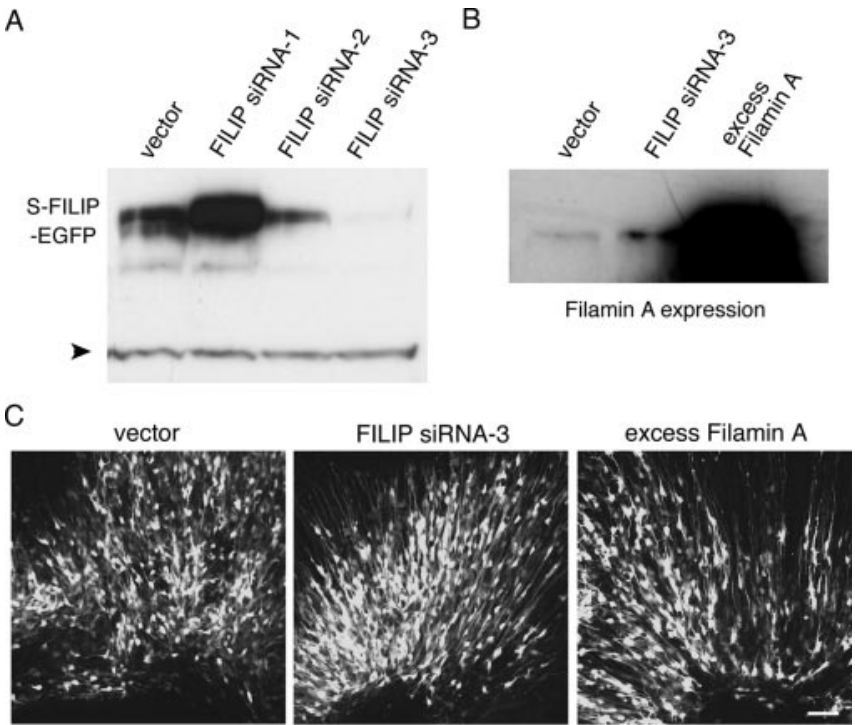

D

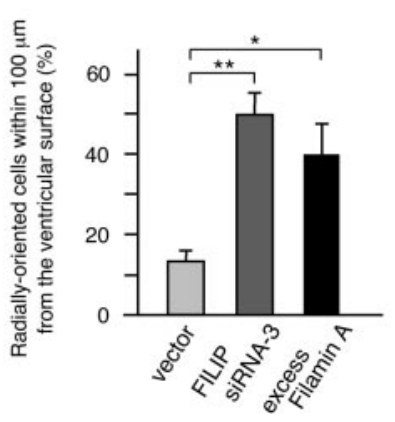

Figure 2. Filamin A determines the shape of neocortical neurons in cultured explants. $A$, The effects of three siRNAs for FILIP, expressed using mU6pro-based vectors, were examined by coexpression with S-FILIP-EGFP in COS-7 cells. The amounts of S-FILIP-EGFP present $2 \mathrm{~d}$ after transfection were detected by immunoblotting using anti-GFP antibody. FILIP siRNA-3 was the most effective, whereas FILIP siRNA-2 had little effect. The mU6pro vector without any additional insert (vector) served as a negative control, whereas nonspecifically reactive bands (arrowhead) served as loading controls. $B$, The amount of Filamin A was assessed by immunoblotting in E18 rat neocortical explants expressing FILIP siRNA-3 or those overexpressing human Filamin A (excess Filamin A) $2 \mathrm{~d}$ after electroporation-mediated gene transfer. Explants transfected with mU6pro vector without additional insert served as a control (vector). FILIP siRNA-3 also caused accumulation of Filamin A as expected. C, The effects of FILIP siRNA-3 or excess Filamin A on cell morphology $2 \mathrm{~d}$ after electroporation-mediated gene transfer into E18 rat neocortical explants were visualized by the coexpression of EGFP. Most labeled cells remained near the ventricular surface on day 2. Most of the cells expressing either FILIP siRNA-3 or excess Filamin A had a similar radially oriented thin spindle shape near the ventricular surface (bottom of each panel). In contrast, most cells transfected with the empty mU6pro vector (vector; negative control) near the ventricular surface were round in shape. Scale bar, $50 \mu \mathrm{m} . D$, The number of radially oriented thin spindle-shaped cells within $100 \mu \mathrm{m}$ from the ventricular surface of each explant was counted, and their percentage relative to the total number of labeled cells in that region was calculated. The mean and SEM for three independent experiments are shown for each group. Asterisks indicate significant difference between the groups $\left({ }^{*} p<0.05\right.$; ${ }^{* *} p<$ $0.01 ;$ t test)

mained near the ventricular surface. We found that many of the FILIP siRNA-3-containing cells near the ventricular surface were radially oriented and spindle shaped; these features were observed less frequently among control cells in the same region (Fig. $2 C$, middle panel, $D$ ). Suppression of FILIP thus alters the shape of neocortical neurons.

To confirm that the increase in radially oriented spindleshaped cells in the FILIP siRNA-3-expressing culture resulted from Filamin A accumulation, we next overexpressed exogenous human Filamin A instead of FILIP siRNA-3. As in the FILIP
siRNA-3-expressing culture, this led to the formation of many radially oriented spindle-shaped cells (Fig. $2 C$, right panel, $D$ ). These results show that the amount of Filamin A, which is regulated in part by FILIP activity, determines neuronal shape during radial migration in organotypic explants.

\section{$\Delta$ ABD-Filamin A inhibits radial migration through the subventricular and intermediate zones to the cortical plate in the developing cerebral cortex in vivo}

The above results suggest that Filamin A is important in regulating the shape and motility of neocortical neurons during radial migration. Therefore, we next investigated the significance of Filamin A in radial migration during neocortical development in vivo. We used in utero electroporation-mediated gene transfer (Inoue and Krumlauf, 2001; Saito and Nakatsuji, 2001; Tabata and Nakajima, 2001), which allows delivery of plasmids into the VZ cells and postmitotic migrating neurons of embryonic neocortices in utero. Because cortices subjected to a sham operation continued to develop normally, this method enabled us to evaluate the net effect of the injected plasmid as much as possible. In this method, we introduced an EGFP or EGFP- $\triangle \mathrm{ABD}$-Filamin A expression plasmid into E15 mouse dorsolateral neocortices in vivo, and labeled cells were observed at E17 (day 2 after transfection) or E19 (day 4 after transfection). On day 2, EGFP- or EGFP$\triangle \mathrm{ABD}$-Filamin A-labeled cells were found mostly in the $\mathrm{VZ}$ and SVZ-IZ (Fig. 3A). We could not detect any inhibitory effect of the mutant Filamin A against the early phase of radial migration up to the SVZ-IZ, because similar percentages of EGFP- $\triangle \mathrm{ABD}$ Filamin A-labeled and EGFP-labeled cells were present outside the VZ (Fig. $3 B$ ). In contrast, these labeled cells showed distinct distribution patterns on day 4 (Fig. $4 A$, top panels, $B$ ). Although a substantial number of EGFP-labeled cells was found in the CP and many of them seemed to have completed radial migration based on their location, most of the EGFP- $\triangle \mathrm{ABD}$-Filamin A-labeled cells were still in the SVZ-IZ. These results suggest that Filamin A is required for radial migration through the SVZ-IZ (or into the $\mathrm{CP}$ ) in vivo.

$\Delta \mathrm{ABD}$-Filamin A causes aberrant regulation of cell polarity in the subventricular and intermediate zones

In the SVZ-IZ of the developing neocortex, many neurons exhibit multipolar migration, during which they do not have any fixed polarity as they do during locomotion or somal translocation in the CP (Tabata and Nakajima, 2003). They move irregularly and slowly, with their multiple neurites extending and retracting dynamically. The finding that mutant Filamin A prevented neuronal migration through the SVZ-IZ therefore prompted us to focus on the neurites of cells in the SVZ-IZ that expressed the mutant.

We observed the morphology of the EGFP- or EGFP- $\triangle \mathrm{ABD}$ Filamin A-expressing cells in the SVZ-IZ on days 2 and 4 after electroporation in utero at E15. On day 2, both the EGFP- and EGFP- $\triangle$ ABD-Filamin A-labeled cells showed multipolar morphology, and their cell bodies and processes tended to be oriented tangentially (Fig. 3A, bottom panels), as described previously (Tabata and Nakajima, 2003). On day 4, the EGFP-labeled cells found in the SVZ-IZ showed a morphology similar to that observed on day 2 (Fig. $4 A$, bottom left panel). In contrast, the EGFP signals from the EGFP-tagged mutant Filamin A-expressing cells on day 4 generally indicated a round shape and a lack of neurites (Fig. $4 \mathrm{~A}$, bottom right panel), suggesting that these cells had lost the neurites that they had possessed $2 \mathrm{~d}$ before. To rule out the possibility that the cells observed on day 4 had 

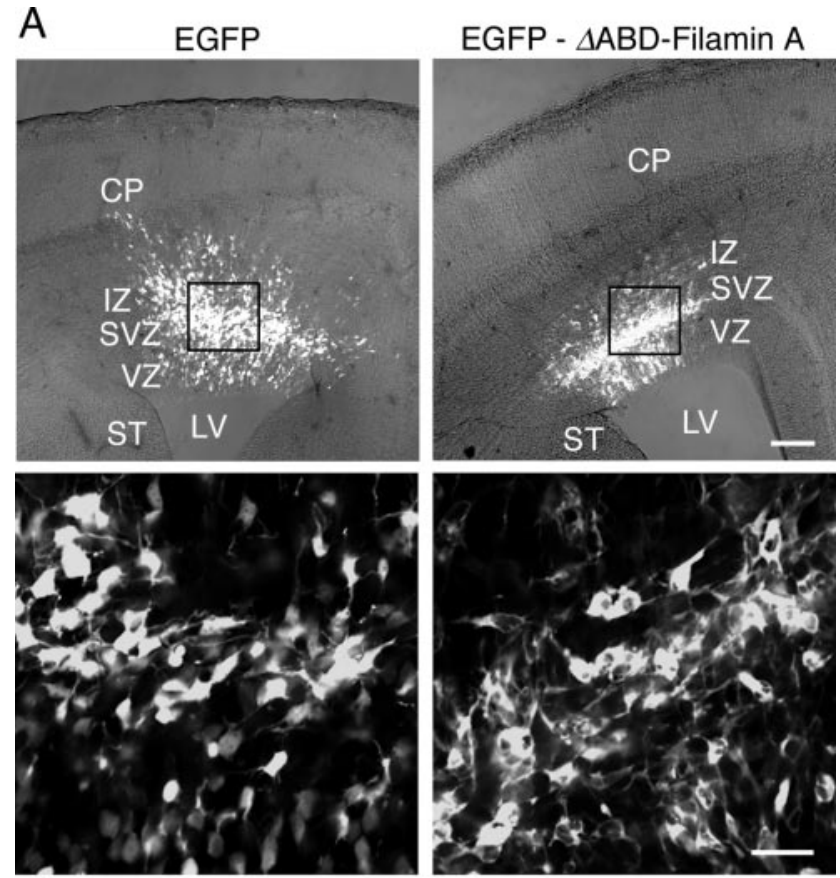

B

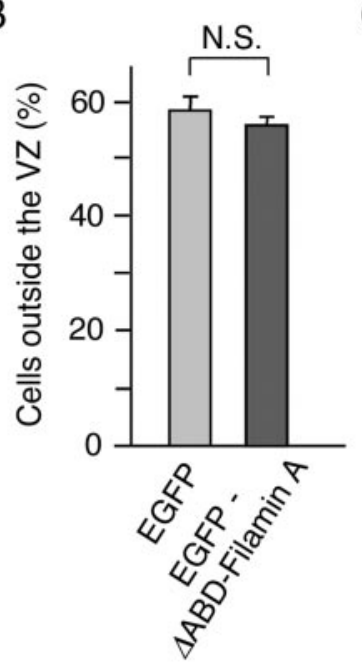

$\mathrm{C} \ll$
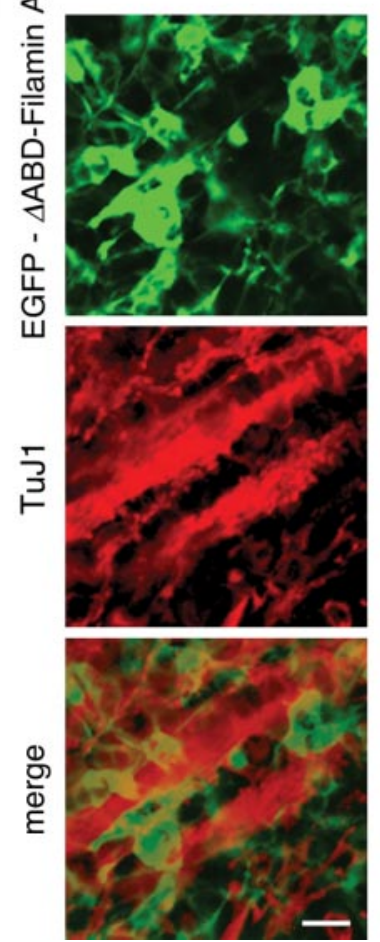

Figure 3. Expression of $\triangle A B D$-Filamin $A$ does not appear to affect cell shape or motility during the early stages of radial migration up to the subventricular or intermediate zone in vivo. $A$, EGFP or EGFP- $\triangle$ ABD-Filamin A was expressed in mouse dorsolateral neocortices in vivo using electroporation-mediated gene transfer on E15. The labeled cells were observed on E17 (day 2). The EGFP- and EGFP- $\triangle$ ABD-Filamin A-labeled cells (left and right columns, respectively) were similar in their distribution and appearance. Difference interference contrast images are merged for the top panels. The bottom panels show higher-magnification views of the areas indicated by the squares in the top panels. LV, Lateral ventricle; ST, striatum. Scale bars: top panels, $100 \mu \mathrm{m}$; bottom panels, $25 \mu \mathrm{m}$. B, Quantitative analyses of cell migration in $A$. The percentages of EGFP- and EGFP- $\triangle A B D$-Filamin A-labeled cells outside the VZ to total number of labeled cells are shown (means and SEM for 3 independent experiments). $t$ test showed that their differences are not significant ( $p>0.05$; NS). C, MostEGFP- $\triangle$ ABD-Filamin A-labeled cells shown in $A$ expressed the neuronal marker TuJ1 (red in middle and bottom panels), as revealed by immunohistochemistry using anti-TuJ1 antibody. Scale bar, $10 \mu \mathrm{m}$.
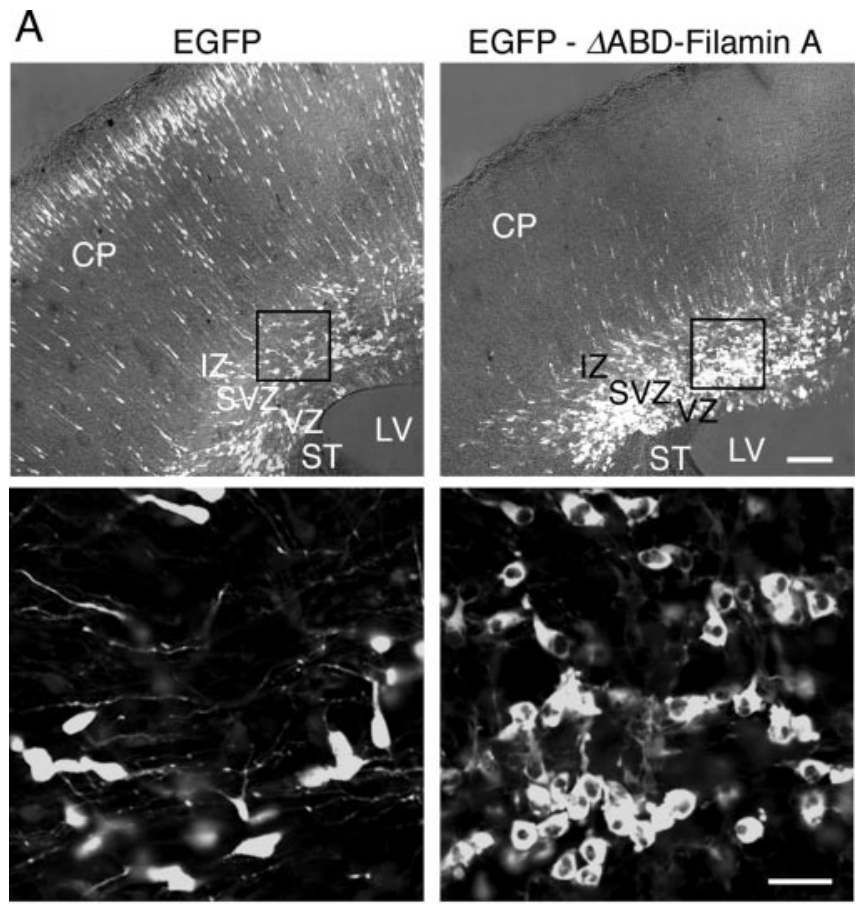

B
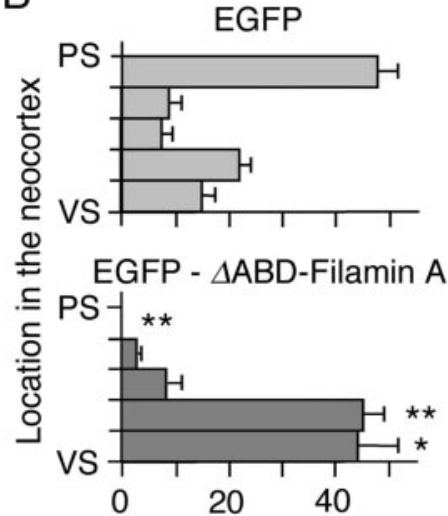

C

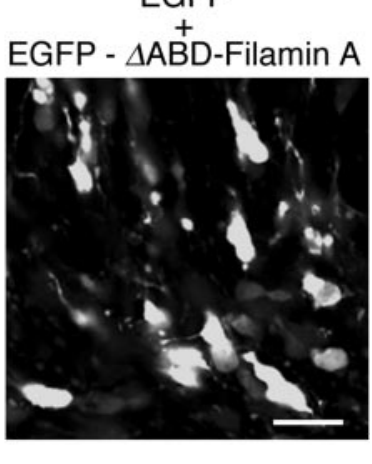

Cells localized in each area (\%)

Figure 4. Filamin $A$ is required for radial migration through the subventricular and intermediate zones in vivo. A, EGFP or EGFP- $\triangle$ ABD-Filamin A was expressed in mouse dorsolateral neocortices in vivo using electroporation-mediated gene transfer on $\mathrm{E} 15$. The labeled cells were observed on E19 (day 4). The EGFP- and EGFP- $\triangle$ ABD-Filamin A-labeled cells (left and right columns, respectively) exhibited different distributions and appearances. Difference interference contrast images are merged for the top panels. The bottom panels show highermagnification views of the areas indicated by the squares in the top panels. Scale bars: top panels, $100 \mu \mathrm{m}$; bottom panels, $25 \mu \mathrm{m}$. B, Quantitative analyses of cell migration in $A$. The neocortex was subdivided evenly into five areas from the VS to the PS. The percentages of labeled cells (cell bodies) in each area were calculated on three independent experiments for each group. Values are means with SEM. Asterisks indicate that these percentages are significantly different from those of the same areas in EGFP-transfected cortices $\left({ }^{*} p<0.05\right.$; ${ }^{* *} p<$ $0.01 ; t$ test). C, EGFP- $\triangle$ ABD-Filamin A was coexpressed with EGFP as in $A$, and the SVZ-IZ cells were observed $4 \mathrm{~d}$ later (on E19). Many of the mutant Filamin A-containing cells in the SVZ-IZ lacked tangentially oriented neurites, which were commonly found in the cells containing only $\operatorname{EGFP}(A$, bottom left panel). The dorsal surface of the neocortex is to the top. Scale bar, $25 \mu \mathrm{m}$.

neurites from which EGFP- $\triangle \mathrm{ABD}$-Filamin A was excluded, we coexpressed EGFP with EGFP- $\triangle$ ABD-Filamin A and observed the SVZ-IZ cells $4 \mathrm{~d}$ later (Fig. 4C). This confirmed that many of the mutant Filamin A-containing cells in the SVZ-IZ lacked tangentially oriented neurites, which were commonly found in the cells containing only EGFP, as shown in Figure $4 A$ (bottom left 
A

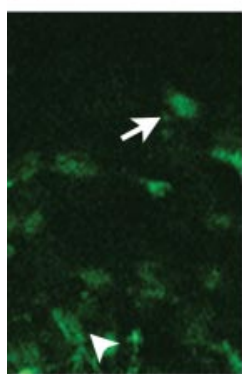

$\mathrm{Oh}$

B

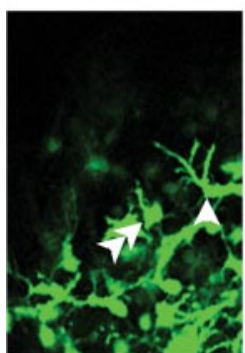

$\mathrm{Oh}$

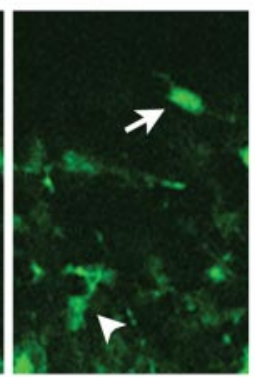

1.0

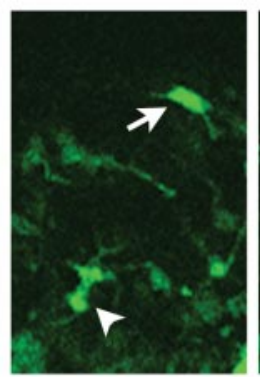

2.0

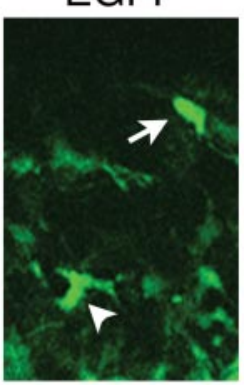

3.0

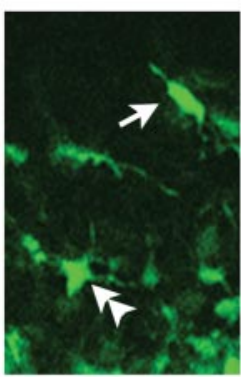

4.0

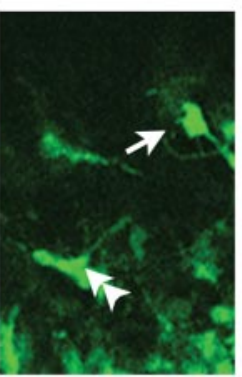

5.0

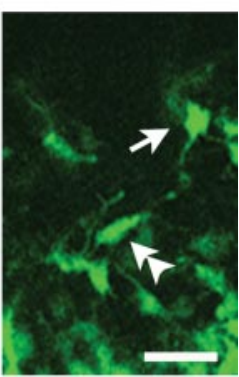

6.0

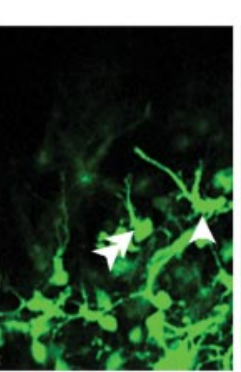

EGFP +

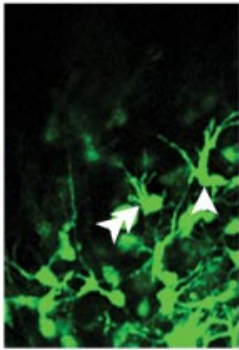

2.0

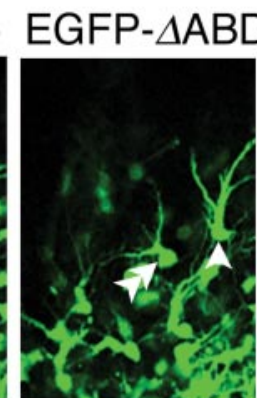

3.0

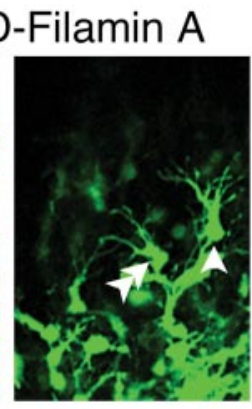

4.0

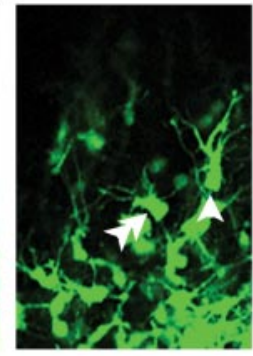

5.0

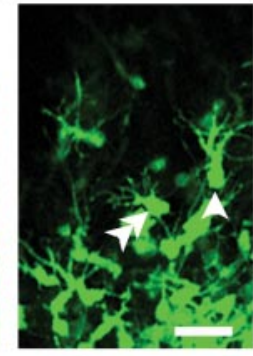

6.0

Figure 5. The antimigratory effect of $\triangle \mathrm{ABD}$-Filamin $\mathrm{A}$ on cells in the intermediate zone is attributable to aberrant regulation of cell polarity. EGFP $(A)$ or EGFP- $\triangle \mathrm{ABD}$-Filamin $\mathrm{A}$ plus EGFP $(B)$ was expressed in mouse dorsolateral neocortices in vivo using electroporation-mediated gene transfer on E15. The labeled cells were analyzed in living slices on E17 (day 2). $A$, The EGFP-expressing cells in the intermediate zone exhibited typical multipolar migration, during which they advanced slowly and irregularly while dynamically extending and retracting multiple processes (arrow and arrowhead). They sometimes took on an almost bipolar shape and temporarily moved faster (double arrowheads; the same cell as indicated by the single arrowhead at 0 - 3.0 hr). $B$, The $\triangle \mathrm{ABD}$-Filamin A-expressing cells also actively and repeatedly extended and retracted multiple neurites; however, the neurites tended to show more complicated branching, and the cells hardly exhibited a bipolar shape or intermittent rapid motion (single and double arrowheads). The pial surface is to the top in each panel. Scale bars, $25 \mu \mathrm{m}$.

panel). In addition, it is unlikely that the mutant Filamin A directly affected their neuronal differentiation, because a substantial fraction of the EGFP- $\triangle \mathrm{ABD}$-Filamin A-labeled cells express neuronal marker TuJ1 (Fig. 3C and data not shown), as reported on EGFP-labeled cells in the SVZ-IZ (Tabata and Nakajima, 2003).

The next question was whether the mutant Filamin A affected neurite dynamics in the SVZ-IZ cells before the neurites were lost and how this effect related to cellular motility. To address these issues, we performed time-lapse observations of cells expressing EGFP-tagged mutant Filamin A plus EGFP or EGFP only in the dorsolateral neocortex $2 \mathrm{~d}$ after in utero electroporation on E15. In the control experiment, during which only EGFP was expressed, many SVZ-IZ cells showed dynamic extension and retraction of multiple neurites in various directions, whereas they migrated slowly and irregularly in a radial direction (Fig. 5A, arrow and arrowhead), as reported previously (Tabata and Nakajima, 2003). In addition, they sometimes took on an almost bipolar shape and temporarily moved faster (Fig. 5A, double arrowheads) (movie $S 1$, supplemental material, available at www. jneurosci.org). The SVZ-IZ cells containing the mutant Filamin A also actively extended and retracted multiple neurites. The neurites tended to show more complicated branching, however, and the cells hardly ever exhibited a bipolar shape or intermittent rapid motion (Fig. 5B, single and double arrowheads) (movie S2, supplemental material, available at www.jneurosci.org). In addition, some of the EGFP- $\triangle \mathrm{ABD}$-Filamin A-labeled cells showed active dynamics of apparently short neurites (an example is found at bottom left in movie S2, supplemental material, available at www.jneurosci.org). The mutant Filamin A therefore seemed to primarily affect cell polarity rather than disrupt neurite dynamics during the cell rounding process in the SVZ-IZ.

Filamin A overexpression caused by FILIP dysfunction also affects cell polarity in the subventricular and intermediate zones

We reported previously that FILIP induces the degradation of Filamin A in VZ cells, in which less Filamin A is accumulated (Nagano et al., 2002). The present results obtained from cultured neocortical explants suggest that the shape of neocortical cells depends on the amount of functional Filamin A present, which is partially regulated by FILIP. Dysfunction of Filamin A makes the neocortical cells round, whereas overexpression induces a spindle-like bipolar shape (Figs. 1, 2C,D). Our in vivo results show that dysfunction of Filamin A affects the regulation of cell polarity, eventually making cells in the SVZ-IZ round (Figs. $4 A$, $5 B)$. These observations led us to hypothesize that the amount of Filamin A present during the early phases of radial migration (i.e., up to the IZ) was responsible for the cell shape during multipolar migration through the SVZ-IZ. To test this, we attempted to increase the Filamin A content of radially migrating cells in vivo by coexpressing FILIP siRNA-3 with EGFP through electroporation in utero on E15. FILIP siRNA-2, which had little effect on FILIP (Fig. 2A), served as a control. First, we examined the amount of Filamin A protein in neocortices expressing these siRNAs on day 2 after electroporation and confirmed that greater accumulation of Filamin A occurred in FILIP siRNA-3expressing than in FILIP siRNA-2-expressing neocortices (Fig. $6 A$ ). We next compared the morphologies of the IZ cells express- 
A

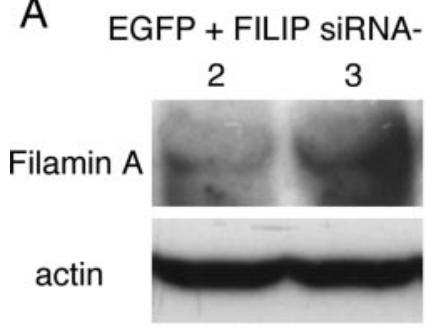

B EGFP + FILIP siRNA-2
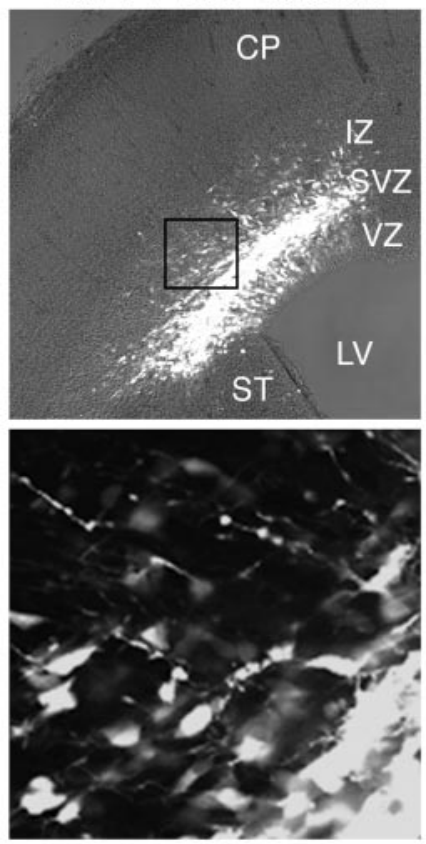

D
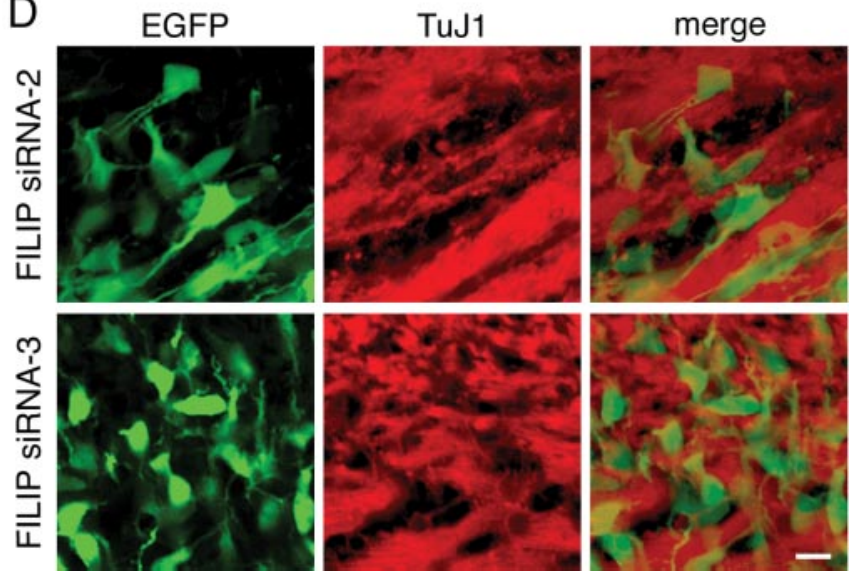

Figure 6. The amount of Filamin A in the intermediate zone cells is important in controlling cell polarity to characterize multipolar migration. FILIP siRNA-2 or -3 was expressed together with EGFP in mouse dorsolateral neocortices in vivo using electroporation-mediated gene transfer on $\mathrm{E} 15$, and the neocortices were analyzed on $\mathrm{E} 17$ (day 2). $A$, Immunoblot analysis confirmed that FILIP siRNA-3 increased Filamin A expression (top panel). FILIP siRNA-2, which had little effect, served as a negative control, whereas expression of actin served as loading controls (bottom panel). $B$, Intermediate zone cells containing FILIP siRNA-3 (right column) tended to show radially oriented spindle shape. Those containing FILIP siRNA-2 (left column) served as controls. Difference interference contrast images are merged for the top panels. The bottom panels show higher-magnification views of the areas indicated by the squares in the top panels. Scale bars: top panels, $100 \mu \mathrm{m}$; bottom panels, $25 \mu \mathrm{m}$. C, Quantitative analyses of cell shape in the intermediate zone. Radially oriented spindle-shaped cells (defined as those with a long axis/short axis ratio of $>2.5$ ) were counted, and their percentage relative to the total number of labeled cells in that region was calculated on three independent experiments in each group. ing these siRNAs on day 2. The results showed that FILIP siRNA-3 caused some of the cells to take on a radial orientation and bipolar spindle shape, whereas multipolar cells typical of those normally seen in the IZ were found predominantly in the neocortices expressing FILIP siRNA-2 (Fig. $6 B, C$ ). On the other hand, neither an apparent change in radial migration out of the VZ up to the IZ based on the locations of the cells (Fig. $6 B$ ) nor in their expression of TuJ1 (Fig. 6D) was noticed on day 2. These results suggest that appropriate expression of Filamin A by cells undergoing radial migration through the SVZ-IZ, which is regulated at least partially by FILIP, is responsible for controlling their polarity to characterize multipolar migration.

Because the radially oriented spindle-shaped cells induced in the IZ by FILIP siRNA-3 showed a morphology similar to those undergoing locomotion or somal translocation in the $\mathrm{CP}$, we next investigated whether their behavior resembled typical migration in the CP. We performed time-lapse observations of IZ cells expressing FILIP siRNA-2 or -3 together with EGFP in the dorsolateral cortex $2 \mathrm{~d}$ after in utero electroporation on E15. The behavior of control cells (those expressing FILIP siRNA-2) was essentially the same as that observed in the neocortices expressing EGFP only (data not shown). In contrast, the IZ cells containing FILIP siRNA-3, which exhibited radially oriented spindle shape, behaved in a distinct manner (Fig. 7, single and double arrowheads) (movie S3, supplemental material, available at www. jneurosci.org). Most of them maintained their radial polarity during the observation period; however, their two major neurites (leading and trailing processes) did not remain still but were repeatedly extended and retracted in the consistent radial directions. Nevertheless, the migration rates of these FILIP siRNA-3-containing bipolar cells were similar to those of FILIP siRNA-2-containing cells [1.33-5.18 $\mu \mathrm{m} / \mathrm{hr}$ (mean 2.44 $\mu \mathrm{m} / \mathrm{hr}$ ) for 10 representative cells in the former group, compared with $0.86-4.50 \mu \mathrm{m} / \mathrm{hr}$ (mean $2.00 \mu \mathrm{m} / \mathrm{hr}$ ) for 9 representative cells in the latter group; $t$ test showed that their differences are not significant $(p>0.05)]$. These results suggest that hyperactivity of Filamin A in the SVZ-IZ cells restricts the cell polarity to radial direction rather than modifying neurite dynamics or cell motility. Therefore, FILIP seems to prevent SVZ-IZ cells from acquiring the fixed polarity to enable multipolar migration.

\section{Discussion}

In the study presented here, we investigated the effect of Filamin A on the radial migration of neocortical neurons in organotypic neocortical explants and the developing neocortex in vivo, in both of which the migration was perturbed and/or observed using an electroporation-mediated gene transfer technique. In the first of these experimental systems, neocortical explants tended to deform as the time spent in vitro culture increased, and the borders between the VZ, SVZ-IZ, and CP became unclear. This made it difficult to establish exact correlations between the behavioral features of the migrating cells in the explants with those found in vivo; however, the findings regarding cell shape and motility were consistent in both systems. $\triangle \mathrm{ABD}$-Filamin $\mathrm{A}$, a

Means with SEM. Double asterisks indicate significant difference between the groups ( $p<$ $0.01 ; t$ test). D, Most intermediate zone cells containing FILIP siRNA-2 or FILIP siRNA-3 shown in $B$ (marked by EGFP; top and bottom panels, respectively) expressed the neuronal marker TuJ1 (red in middle and right columns), as revealed by immunohistochemistry using anti-TuJ1 antibody. Scale bar, $10 \mu \mathrm{m}$. 


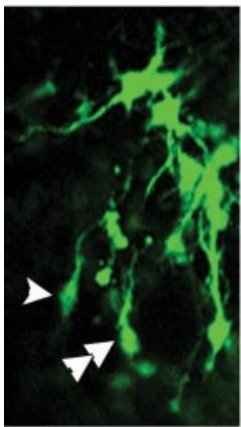

$\mathrm{Oh}$

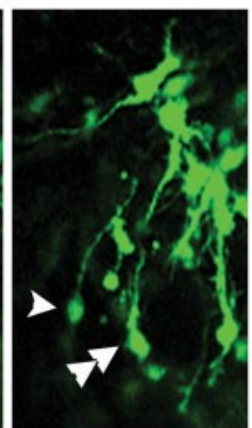

1.0

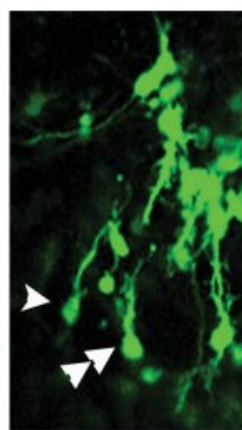

2.0
EGFP + FILIP siRNA-3

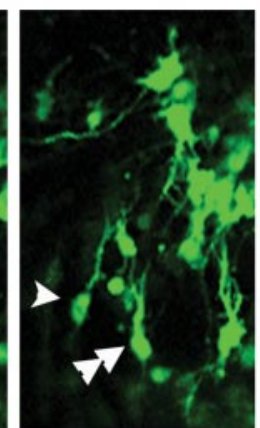

3.0

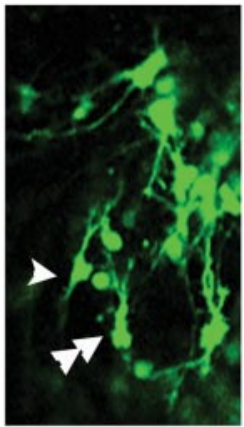

4.0

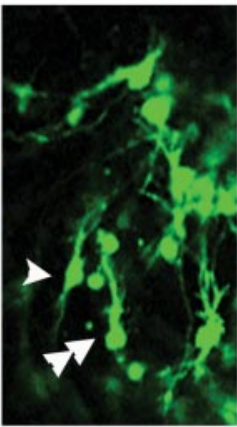

5.0

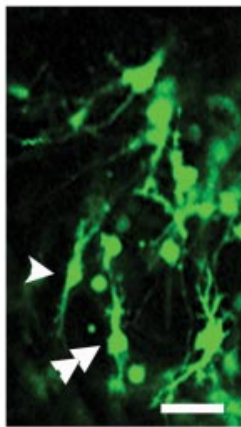

6.0

Figure 7. Overexpression of Filamin A in the cells of the intermediate zone restricts cell polarity to radial direction, rather than modifying neurite dynamics or cell motility. FILIP siRNA-3 was coexpressed with EGFP in mouse dorsolateral neocortices in vivo using electroporation-mediated gene transfer on E15. The labeled cells were analyzed in living slices on E17 (day 2). Many FILIP siRNA-3-expressing cells in the intermediate zone maintained their polarity during the observation period, unlike control cells, which exhibited multipolar migration. However, they repeatedly extended and retracted their neurites in consistently radial directions (arrowhead and double arrowheads) and showed motility rates similar to cells containing FILIP siRNA-2 (see Results). The pial surface is to the top in each panel. Scale bar, $25 \mu \mathrm{m}$.

dominant-negative mutant that inhibits the function of normal Filamin A (Kainulainen et al., 2002), caused the cells to become round, produce few neurites, and become less motile, whereas Filamin A overexpression caused by an effective FILIP siRNA promoted a radially oriented spindle shape. The data from both experimental systems thus indicate that Filamin A and FILIP act on cell polarity and motility in migrating neocortical neurons. In addition, the results from the in vivo experimental system revealed that these Filamin A functions significantly affect multipolar cells in the SVZ-IZ. Time-lapse observations further suggested that Filamin A and FILIP help to determine whether the SVZ-IZ cells are multipolar or bipolar (i.e., spindle-shaped). According to Tabata and Nakajima (2003), the SVZ-IZ of the developing neocortex can be characterized by abundant multipolar cells that have no fixed polarity but dynamically extend and retract their neurites. These cells contrast markedly with those migrating in the $\mathrm{CP}$, which typically exhibit somal translocation or locomotion in a constant monopolar or bipolar form (Miyata et al., 2001; Nadarajah et al., 2001). Our results suggest for the first time that the regulation of cell polarity in the SVZ-IZ by Filamin A could be the molecular basis of multipolar migration. Overexpression of Filamin A restricts cell polarity to a radial direction, whereas its dysfunction prevents the cells from acquiring fixed polarity. Normal Filamin A activity, which is regulated at least partially by FILIP, thus enables multipolar migration through coordinating cell polarity. The shape of the SVZ-IZ cells can be affected by such cell-autonomous factors as FILIP and Filamin A, regardless of external cues (e.g., contact with radial cells and/or extracellular matrices, etc.). This does not necessarily imply that the SVZ-IZ cells regulate their shape autonomously. As will be argued in the last paragraph of Discussion, Filamin A might regulate recognition of external cues to make the cells bipolar that otherwise tend to be autonomously multipolar. Our results also show that Filamin A activity is not likely a sole determinant for migration mode. Ectopic bipolar cells induced by Filamin A overexpression in the SVZ-IZ do not show normal locomotion or somal translocation. They migrate as slowly as multipolar migration rather than normal locomotion or somal translocation in the $\mathrm{CP}$, suggesting that some additional factors are required for normal transition of the migration mode at the IZ-CP border (i.e., from multipolar migration to locomotion or somal transloca- tion). For example, environmental cues specific to the CP might be involved to adjust the migration speed appropriately in the CP. During the migration through the SVZ-IZ, in contrast, regulation of cell shape and that of migration speed are independent with each other.

We reported previously that FILIP, the gene expression of which is restricted to the VZ in the developing neocortex, appears to induce the degradation of Filamin A in the $\mathrm{VZ}$ and regulate the start of radial migration (Nagano et al., 2002). Our present results clearly show the significance of both FILIP and Filamin A in cells migrating through the SVZ-IZ; however, we cannot exclude the possibility that they were also required for normal cell behavior in the $\mathrm{VZ}$ and/or the appropriate starting of migration from the $\mathrm{VZ}$ based on the present results, because of the time lag between gene transfer into the VZ cells and accumulation of sufficient mutant Filamin A or FILIP siRNA to reveal their effects. It is likely that a substantial number of the transfected cells started migration from the VZ before they accumulated enough of the mutant or the siRNA.

Our present findings serve to provide greater understanding of the pathogenesis of periventricular nodular heterotopia, a human neocortical malformation caused by various loss-offunction mutations in the filamin A gene (Fox et al., 1998; Sheen et al., 2001; Kakita et al., 2002). In this disorder, neurons lacking normal Filamin A are thought to accumulate beside the lateral ventricle (Eksioglu et al., 1996; Kakita et al., 2002). In our in vivo experiments in the mouse, most of the neurons overexpressing the mutant Filamin A remained in the SVZ-IZ and not always beside the lateral ventricle on E19 (day 4 after transfection) (Fig. $4 A$, bottom right panel). Therefore, our results suggest a novel possibility that Filamin A is required for radial migration through SVZ-IZ. This is still consistent with the development of the human malformation, because the VZ and SVZ disappear as the brain matures, and most neurons that become mislocated as shown in our in vivo experiment will be positioned beside the lateral ventricle. This is similar to what is observed with doublecortin gene-knock-down neurons, which are located in the IZ early in development (Bai et al., 2003). Of course we cannot exclude the possibility that we may not be able to sufficiently estimate the earlier phases of radial migration using the present methods as discussed above. On the other hand, it should be 
noted that Filamin A can form heterodimers with Filamin B, a highly homologous protein to Filamin A (Takafuta et al., 1998), and that their interaction potentially compensates for the loss of Filamin A function in the development of periventricular nodular heterotopia (Sheen et al., 2002). Then, the mutant Filamin A used in our study is also likely to form heterodimer with endogenous Filamin B because our mutant Filamin A has an intact C-terminal domain through which Filamin A and B interact with each other. Therefore, it is possible that the mutant Filamin A suppresses compensation by the Filamin A/B heterodimer as well as the function of Filamin A, leading to more severe perturbations than those found in human malformation. This might be one of the reasons that the cells with the mutant Filamin A in our results were lacking in neurites on day 4 , whereas mislocated neurons in the periventricular nodular heterotopia without functional Filamin A have almost normal morphology in the adult stage (Eksioglu et al., 1996; Kakita et al., 2002).

Filamin A is known to interact with various proteins, including cell-surface receptors and signal transduction molecules (Stossel et al., 2001). Among these, several possible candidates that may link Filamin A with cell polarity and motility in neocortical neurons have recently emerged, although whether they are authentically present in neocortical neurons has not yet been confirmed. For example, Bellanger et al. (2000) reported that Filamin interacts with the Rac1-activating protein Trio and controls its intracellular distribution in HeLa cells. Because other Racl-activators, Sif and Tiam1-like exchange factor (STEF)/Tiam1, and activated Rac1 itself are required for radial migration through the IZ (Kawauchi et al., 2003), it is possible that Trio cooperates with STEF/Tiam1 to activate Racl in neocortical neurons and that Filamin A specifies the whereabouts within the cell to activate Racl to promote multipolar migration through the IZ. Therefore, it is particularly interesting that Kawauchi et al. (2003) found that IZ cells containing a dominant-negative Rac1 mutant exhibit a round shape, similar to the cells containing mutant Filamin A during our observation. Is the radially oriented spindle shape, which results from Filamin A overexpression, also observed in IZ cells with Rac1 and/or STEF/Tiam1 hyperactivity? Is the activity of Racl suppressed in IZ cells containing mutant Filamin A? Further studies to address these questions could provide us with possible clues to clarify whether Racl acts at the downstream of Filamin A. Alternatively, Migfilin, which links the cell-matrix adhesion protein Mig-2 with Filamin A and actin cytoskeleton ( $\mathrm{Tu}$ et al., 2003), might be another candidate. Because it has been demonstrated that loss of either Migfilin or Mig-2 impairs cell shape in cultured cells, it is possible that these molecules link Filamin A to regulation of neocortical cell shape or polarity during radial migration if they are also expressed endogenously. Of course it is still possible, however, that the downstream molecules of Filamin A affect intercellular interaction rather than intracellular machinery to regulate cell polarity and shape directly. The radially oriented bipolar cells in the SVZ-IZ caused by Filamin A overexpression might be a consequence of increased affinity with a radial cell. The fact that Filamin A is capable of interacting with and modifying the functions of many cell-surface molecules (Stossel et al., 2001) supports this possibility.

\section{References}

Bai J, Ramos RL, Ackman JB, Thomas AM, Lee RV, LoTurco JJ (2003) RNAi reveals doublecortin is required for radial migration in rat neocortex. Nat Neurosci 6:1277-1283.
Bellanger JM, Astier C, Sardet C, Ohta Y, Stossel TP, Debant A (2000) The Rac1- and RhoG-specific GEF domain of Trio targets filamin to remodel cytoskeletal actin. Nat Cell Biol 2:888-892.

Eksioglu YZ, Scheffer IE, Cardenas P, Knoll J, DiMario F, Ramsby G, Berg M, Kamuro K, Berkovic SF, Duyk GM, Parisi J, Huttenlocher PR, Walsh CA (1996) Periventricular heterotopia: an X-linked dominant epilepsy locus causing aberrant cerebral cortical development. Neuron 16:77-87.

Feng Y, Olson EC, Stukenberg PT, Flanagan LA, Kirschner MW, Walsh CA (2000) LIS1 regulates CNS lamination by interacting with mNudE, a central component of the centrosome. Neuron 28:665-679.

Fox JW, Lamperti ED, Eksioglu YZ, Hong SE, Feng Y, Graham DA, Scheffer IE, Dobyns WB, Hirsch BA, Radtke RA, Berkovic SF, Huttenlocher PR, Walsh CA (1998) Mutations in filamin 1 prevent migration of cerebral cortical neurons in human periventricular heterotopia. Neuron 21:1315-1325.

Gupta A, Tsai LH, Wynshaw-Boris A (2002) Life is a journey: a genetic look at neocortical development. Nat Rev Genet 3:342-355.

Hatanaka Y, Murakami F (2002) In vitro analysis of the origin, migratory behavior, and maturation of cortical pyramidal cells. J Comp Neurol 454:1-14.

Inoue T, Krumlauf R (2001) An impulse to the brain-using in vivo electroporation. Nat Neurosci [Suppl] 4:1156-1158.

Kainulainen T, Pender A, D'Addario M, Glogauer M, Lekic P, McCulloch CA (2002) Cell death and mechanoprotection by filamin A in connective tissues after challenge by applied tensile forces. J Biol Chem 277:21998-22009.

Kakita A, Hayashi S, Moro F, Guerrini R, Ozawa T, Ono K, Kameyama S, Walsh CA, Takahashi H (2002) Bilateral periventricular nodular heterotopia due to filamin 1 gene mutation: widespread glomeruloid microvascular anomaly and dysplastic cytoarchitecture in the cerebral cortex. Acta Neuropathol (Berl) 104:649-657.

Kawauchi T, Chihama K, Nabeshima Y, Hoshino M (2003) The in vivo roles of STEF/Tiam1, Rac1 and JNK in cortical neuronal migration. EMBO J 22:4190-4201.

Miyata T, Kawaguchi A, Okano H, Ogawa M (2001) Asymmetric inheritance of radial glial fibers by cortical neurons. Neuron 31:727-741.

Nadarajah B, Brunstrom JE, Grutzendler J, Wong RO, Pearlman AL (2001) Two modes of radial migration in early development of the cerebral cortex. Nat Neurosci 4:143-150.

Nagano T, Yoneda T, Hatanaka Y, Kubota C, Murakami F, Sato M (2002) Filamin A-interacting protein (FILIP) regulates cortical cell migration out of the ventricular zone. Nat Cell Biol 4:495-501.

Niwa H, Yamamura K, Miyazaki J (1991) Efficient selection for high-expression transfectants with a novel eukaryotic vector. Gene 108:193-199.

Noctor SC, Martinez-Cerdeno V, Ivic L, Kriegstein AR (2004) Cortical neurons arise in symmetric and asymmetric division zones and migrate through specific phases. Nat Neurosci 7:136-144.

Rakic P (1972) Mode of cell migration to the superficial layers of fetal monkey neocortex. J Comp Neurol 145:61-83.

Rakic P, Knyihar-Csillik E, Csillik B (1996) Polarity of microtubule assemblies during neuronal cell migration. Proc Natl Acad Sci USA 93:9218-9222.

Rivas RJ, Hatten ME (1995) Motility and cytoskeletal organization of migrating cerebellar granule neurons. J Neurosci 15:981-989.

Saito T, Nakatsuji N (2001) Efficient gene transfer into the embryonic mouse brain using in vivo electroporation. Dev Biol 240:237-246.

Schaar BT, Kinoshita K, McConnell SK (2004) Doublecortin microtubule affinity is regulated by a balance of kinase and phosphatase activity at the leading edge of migrating neurons. Neuron 41:203-213.

Sheen VL, Dixon PH, Fox JW, Hong SE, Kinton L, Sisodiya SM, Duncan JS, Dubeau F, Scheffer IE, Schachter SC, Wilner A, Henchy R, Crino P, Kamuro K, DiMario F, Berg M, Kuzniecky R, Cole AJ, Bromfield E, Biber M, et al. (2001) Mutations in the X-linked filamin 1 gene cause periventricular nodular heterotopia in males as well as in females. Hum Mol Genet 10:1775-1783.

Sheen VL, Feng Y, Graham D, Takafuta T, Shapiro SS, Walsh CA (2002) Filamin A and Filamin B are co-expressed within neurons during periods of neuronal migration and can physically interact. Hum Mol Genet 11:2845-2854.

Smith DS, Niethammer M, Ayala R, Zhou Y, Gambello MJ, WynshawBoris A, Tsai LH (2000) Regulation of cytoplasmic dynein behaviour and microtubule organization by mammalian Lis1. Nat Cell Biol 2:767-775. 
Stossel TP, Condeelis J, Cooley L, Hartwig JH, Noegel A, Schleicher M, Shapiro SS (2001) Filamins as integrators of cell mechanics and signaling. Nat Rev Mol Cell Biol 2:138-145.

Tabata H, Nakajima K (2001) Efficient in utero gene transfer system to the developing mouse brain using electroporation: visualization of neuronal migration in the developing cortex. Neuroscience 103:865-872.

Tabata H, Nakajima K (2003) Multipolar migration: the third mode of radial neuronal migration in the developing cerebral cortex. J Neurosci 23:9996-10001.

Takafuta T, Wu G, Murphy GF, Shapiro SS (1998) Human beta-filamin is a new protein that interacts with the cytoplasmic tail of glycoprotein Ibalpha. J Biol Chem 273:17531-17538.
Tanaka T, Serneo FF, Tseng HC, Kulkarni AB, Tsai LH, Gleeson JG (2004) Cdk5 phosphorylation of Doublecortin Ser297 regulates its effect on neuronal migration. Neuron 41:215-227.

Tu Y, Wu S, Shi X, Chen K, Wu C (2003) Migfilin and Mig-2 link focal adhesions to filamin and the actin cytoskeleton and function in cell shape modulation. Cell 113:37-47.

Xie Z, Sanada K, Samuels BA, Shih H, Tsai LH (2003) Serine 732 phosphorylation of FAK by Cdk5 is important for microtubule organization, nuclear movement, and neuronal migration. Cell 114:469-482.

Yu JY, DeRuiter SL, Turner DL (2002) RNA interference by expression of short-interfering RNAs and hairpin RNAs in mammalian cells. Proc Natl Acad Sci USA 99:6047-6052. 